\title{
Subtracting Out Complex Singularities in Numerical Integration
}

\author{
By F. G. Lether
}

\begin{abstract}
This paper is concerned with the numerical approximation of definite integrals over $[-1,1]$, in which the function $f$ to be integrated has isolated singularities near $[-1,1]$. Complex variable techniques are used to study the effectiveness of the method of subtracting out complex singularities.
\end{abstract}

1. Introduction. This paper deals with the approximation of integrals of the form

$$
I(f)=\int_{-1}^{1} \omega(x) f(x) d x
$$

where $\omega$ is a given weight function.

When $f$ is well behaved, standard quadrature techniques often produce accurate approximations to $I(f)$ using relatively few evaluations of $f$. However, it is well known that singularities of $f$ in the complex plane near $[-1,1]$, may have adverse effects on the accuracy of the commonly employed numerical integration methods.

In this paper, a method is presented for approximating $I(f)$ when $f$ has isolated complex singularities near $[-1,1]$. The idea employed is essentially an extension into the complex domain of the method of subtracting out real singularities found in [1] and [2, p. 202]. An error analysis is given that determines conditions under which this technique is effective.

2. Preliminaries. Let

$$
I(f)=Q_{n}(f)+R_{n}(f)
$$

where

$$
Q_{n}(f)=\sum_{k=1}^{n} w_{k} f\left(x_{k}\right)
$$

be a given quadrature rule. Assume that (2.1) has precision $d$ and that $x_{k} \in[-1,1]$.

We would expect $I(f) \approx Q_{n}(f)$ to be a good approximation for nonpolynomial functions $f$, provided $f$ can be accurately approximated on $[-1,1]$ by a polynomial of degree $\leqslant d$. This is generally not the case when $f$ has singularities in the complex plane near $[-1,1]$. Therefore, it is reasonable to consider the possibility of subtracting from $f$ some function $s$, so that $f-s$ has no singularities near $[-1,1]$.

Received August 25, 1975 ; revised May 13, 1976.

AMS (MOS) subject classifications (1970). Primary 65D30.

Key words and phrases. Complex singularities, quadrature, subtracting out singularities.

Copyright 1977. American Mathematical Society 
By (2.1) and the identity

$$
f(x)=s(x)+[f(x)-s(x)]
$$

we can write

$$
I(f)=I(s)+Q_{n}(\varphi)+R_{n}(\varphi)
$$

where

$$
\varphi(x)=f(x)-s(x)
$$

Suppose that a function $s$ can be determined so that a closed form for $I(s)$ is known, and that $\varphi$ resembles a polynomial. Then we may think of the right side of (2.2) as consisting of the following components: a known definite integral; a quadrature rule evaluation of $\varphi=f-s$; a remainder term.

3. Subtracting Out Isolated Singularities. Let $f(z)$ denote the analytic continuation of $f(x)$ into the complex $z$-plane. Assume that $f(z)$ is analytic in the finite $z$ plane, except for isolated complex singularities at $a_{j} \notin[-1,1], j=1,2,3, \ldots$.

Consider the $m$ singularities $a_{j}, 1 \leqslant j \leqslant m$, of $f$ near $[-1,1]$. For each $j$, let $\tau_{j}$ and the constants $b_{\nu, j}$ be given, and introduce the function

$$
P_{j}(x)=\sum_{\nu=1}^{\tau_{j}} b_{\nu, j}\left(x-a_{j}\right)^{-\nu}, \quad 1 \leqslant j \leqslant m .
$$

Finally, set

$$
s(x)=\sum_{j=1}^{m} P_{j}(x)
$$

and as before, define $\varphi$ by

$$
f(x)=\sum_{j=1}^{m} P_{j}(x)+\varphi(x)
$$

Then (2.2) becomes

$$
I(f)=-\sum_{j=1}^{m} \sum_{\nu=1}^{\tau_{j}} b_{\nu, j} T^{(\nu-1)}\left(a_{j}\right) /(\nu-1) !+Q_{n}(\varphi)+R_{n}(\varphi),
$$

where we require a closed expression for

$$
T(z)=\int_{-1}^{1} \frac{\omega(x)}{z-x} d x, \quad z \notin[-1,1]
$$

(See Section 5.)

Although (3.2) is valid for any choice of $m, \tau_{j}$ and the $b_{\nu, j}$ 's, their selection influences the accuracy of the method. As a simple illustration, let

$$
f(x)=q_{N}(x)\left(x-a_{1}\right)^{-2}\left(x-a_{2}\right)^{-2},
$$

where $q_{N}$ is a polynomial of degree $N>4$ and $a_{1}, a_{2} \notin[-1,1]$. Take $m=\tau_{1}=$ $\tau_{2}=2$ and let $b_{1, j}$ and $b_{2, j}$ be the Laurent coefficients in the principal part of $f$ 
about the second order pole $a_{j}, j=1,2$. This choice forces $\varphi$ to be a polynomial of degree $N-4$. If the precision of (2.1) satisfies $d \geqslant N-4$, then $R_{n}(\varphi)=0$ and (3.2) becomes

$$
I(f)=-\sum_{j=1}^{2}\left[b_{1, j} T\left(a_{j}\right)+b_{2, j} T^{\prime}\left(a_{j}\right)\right]+Q_{n}(\varphi) .
$$

The work required to compute the Laurent coefficients is repaid, since $Q_{n}(\varphi)$ is more accurate than $Q_{n}(f)$.

Unless $f$ is a rational function, as in (3.3), it is not always possible to choose the parameters $m, \tau_{j}$ and the $b_{\nu, j}$ 's in (3.2) to make $R_{n}(\varphi)=0$. However, for general functions $f$ we can make $R_{n}(\varphi)$ negligible in certain cases by taking $s$ to be the sum of the principal parts of $f$ at its nearby singularities, as was done in the previous example. For this choice of $s, \varphi$ has no nearby singularities and Gaussian rules can profitably be used for (2.1) to maximize $d$. This technique is particularly effective when $f$ is a meromorphic function having only a few low order poles. For example [4], consider the numerical approximation of

$$
I_{1}(f)=\int_{-1}^{1}\left(x^{2}+10^{-4}\right)^{-1} e^{x} d x
$$

by (3.2). The integrand has simple poles at $a_{1}=0.01 i$ and $a_{2}=\bar{a}_{1}$. Take $\omega(x) \equiv 1$, $m=2$ and $\tau_{1}=\tau_{2}=1$ to account for both of the poles. The required residues are

$$
b_{1,1}=\operatorname{Res}_{z=a_{1}} f(z)=-50 i \exp (0.01 i)
$$

and $b_{1,2}=\bar{b}_{1,1} \cdot(3.2)$ gives

$$
I_{1}(f)=I_{1}(s)+Q_{n}(\varphi)+R_{n}(\varphi)
$$

where

$$
I_{1}(s)=-2 \operatorname{Re}\left\{b_{1,1} T\left(a_{1}\right)\right\}
$$

and $T(z)=\ln [(z+1) /(z-1)]$. For the basic generating rule $(2.1)$, we use the $n$ point Gauss-Legendre rule. Table 1 contains the Gaussian quadrature results $Q_{n}(f)$ and the improved approximation $I_{1}(s)+Q_{n}(\varphi)$.

\section{TABLE 1}

\begin{tabular}{rrr}
\hline$n$ & $Q_{n}(f)$ & $I_{1}(s)+Q_{n}(\varphi)$ \\
\hline 2 & 7.02 & 313.171804022 \\
3 & 8891.32 & 313.172055084 \\
4 & 13.24 & 313.172056236 \\
& & $I_{1}=313.172056239$ \\
\hline
\end{tabular}

The superiority of (3.2) over the Gauss-Legendre rule (2.1) is quite evident in this case. 
4. Error Analysis. The idea of subtracting off singularities exactly, so that $R_{n}(\varphi)=0$, is in theory not restricted to the case when $f$ is a rational function. In the following work we seek other functions $f$ for which $R_{n}(\varphi)=0$.

To discuss the effect the singularities $a_{j}$ have on the numerical approximation of $I(f)$, we introduce the following contour. Let $\Gamma$ be a simple, closed, rectifiable contour which contains $[-1,1]$ in its interior, $\Gamma_{i}$. Assume that the only singularities in $\Gamma_{i}-[-1$, 1] are $a_{j}, 1 \leqslant j \leqslant m$.

Let (3.1) be the principal part of $f$ at $a_{j}$. If $a_{j}$ is an essential singularity, then $\tau_{j}=\infty$ and (3.1) is an infinite series. Since $a_{j} \notin[-1,1],(3.1)$ is absolutely and uniformly convergent for $z=x \in[-1,1]$. Of course, this is immediate in the case when $a_{j}$ is a pole of order $\tau_{j}$, since (3.1) is then a finite sum. In the latter case the Laurent coefficients can be expressed in the form

$$
b_{\nu, j}=\frac{1}{(\tau-\nu) !} \lim _{z \rightarrow a_{j}} \frac{d^{\tau-\nu}}{d z^{\tau-\nu}}\left[\left(z-a_{j}\right)^{\tau} f(z)\right],
$$

$\nu=1,2, \ldots, \tau$, where $\tau=\tau_{j}$.

THEOREM 1. Under the previous assumptions on $\Gamma$ and (3.1), the quadrature error in (3.2) is given by

$$
R_{n}(\varphi)=\frac{1}{2 \pi i} \int_{\Gamma} k_{n}(z) f(z) d z,
$$

where the kernel function $k_{n}(z)=R_{n}\left[(z-x)^{-1}\right]$.

If

$$
\sigma=\min _{z \in \Gamma}|z|>1,
$$

then

$$
\left|R_{n}(\varphi)\right| \leqslant e_{n} l(\Gamma) M_{\Gamma}(f) \sigma^{-d-2}\left(1-\sigma^{-1}\right)^{-1} / 2 \pi,
$$

where

$$
l(\Gamma)=\text { length of } \Gamma, \quad e_{n}=\sup _{\nu \geqslant d+1}\left|R_{n}\left(x^{\nu}\right)\right|<\infty
$$

and

$$
M_{\Gamma}(f)=\max _{z \in \Gamma}|f(z)| .
$$

Proof. For $x \in[-1,1]$ the residue theorem yields

$$
\begin{aligned}
\frac{1}{2 \pi i} \int_{\Gamma}(z-x)^{-1} f(z) d z & =f(x)+\sum_{j=1}^{m} \operatorname{Res}_{z=a_{j}}\left[(z-x)^{-1} f(z)\right] \\
& =f(x)-\sum_{j=1}^{m} P_{j}(x)=\varphi(x) .
\end{aligned}
$$

The linear functional $R_{n}$ can be moved through the integral sign in (4.3) to obtain (4.1).

For $|z|>1$, the series

$$
(z-x)^{-1}=\sum_{\nu \geqslant 0} x^{\nu} z^{-\nu-1}
$$


is uniformly convergent on $-1 \leqslant x \leqslant 1$. Since (2.1) has precision $d$,

$$
k_{n}(z)=\sum_{\nu \geqslant d+1} R_{n}\left(x^{\nu}\right) z^{-\nu-1}, \quad|z|>1
$$

Therefore,

$$
\left|k_{n}(z)\right| \leqslant e_{n} \sum_{\nu \geqslant d+1} \sigma^{-\nu-1}=e_{n} \sigma^{-d-2}\left(1-\sigma^{-1}\right)^{-1}
$$

for $z \in \Gamma$. (4.2) follows directly from this inequality and (4.1). This completes the proof.

Stenger [5] has tabulated the constant $e_{n}$ for several of the Gaussian rules and uses the notation $\nu(n)$ in place of $e_{n}$.

The inequality (4.2) has an interesting application. Take $\Gamma=\Gamma_{r}=\{z:|z|=r\}$. Suppose we let $r \rightarrow \infty$ in such a way that no singularity $a_{j} \in \Gamma_{r}$, and assume for this sequence of radii there exists a constant $c$, independent of $r$, such that

$$
\max _{|z|=r}|f(z)| \leqslant c r^{d}
$$

By (4.2) and (4.4),

$$
\left|R_{n}(\varphi)\right| \leqslant c e_{n} r^{-1}\left(1-r^{-1}\right)^{-1}
$$

Since the right side of (4.5) approaches zero as $r \rightarrow \infty$, we have the following result.

COROllary 1. The rule

$$
I(f)=-\sum_{j \geqslant 1} \sum_{\nu=1}^{\tau_{j}} b_{\nu, j} T^{(\nu-1)}\left(a_{j}\right) /(\nu-1) !+Q_{n}(\varphi)
$$

is exact for all functions $f$ satisfying (4.4), where the summation on $j$ extends over all isolated singularities of $f$.

McNamee [3, p. 379] has previously considered the special case of (4.6) when $f$ has simple poles and (2.1) is the $n$-point Gauss-Legendre rule.

It follows from Corollary 1 that (4.6) is exact whenever $f$ is a rational function, provided the precision $d$ of $(2.1)$ is sufficiently large. This agrees with our comments regarding (3.4). However, (4.6) is not restricted to rational functions. For example, it holds for the meromorphic function $(x+2)^{-2}(\sin 10 \pi x-1.1)^{-1}$, which has an infinite number of poles, and the function $(x+\lambda)^{-2} \exp \left[(x+\lambda)^{-1}\right], \lambda>1$. The latter function has an essential singularity at $-\lambda$.

That (4.6) is exact for certain functions having an infinite number of isolated singularities, or an essential singularity, is mainly of theoretical interest. In practice, we cannot subtract out the singularities exactly to make $R_{n}(\varphi)=0$, because $s$ contains an infinite number of terms in these cases. The goal is to subtract off as much of the singular part of $f$ as practical, in order that $\varphi=f-s$ resemble, but not necessarily coincide with a polynomial.

5. Hilbert Transforms. In applications of (3.2) it is convenient to have a closed form for the Hilbert transform $T(z)$. These can be worked out for several of the commonly encountered weight functions. 
Case 1. $T(z)=\int_{-1}^{1}|x|^{m}(z-x)^{-1} d x$.

$$
T(z)=z^{m} \ln \frac{z+1}{z-1}-2 \sum_{k=0}^{t-1} z^{m-1-2 k} /(2 k+1) \quad \text { if } m=2 t,
$$

and

$$
T(z)=z^{m} \ln \frac{z^{2}}{z^{2}-1}-\sum_{k=1}^{t} z^{m-2 k} / k \quad \text { if } m=2 t+1,
$$

$m=1,2,3, \ldots$. The second summation is defined as zero when $t=0$.

Case 2. $T(z)=\int_{0}^{1} x^{m-1 / 2}(z-x)^{-1} d x$.

$$
T(z)=-(\sqrt{z})^{2 m-1} \ln \frac{\sqrt{z}-1}{\sqrt{z}+1}-2 \sum_{k=0}^{m-1} z^{k} /(2 m-2 k-1),
$$

$m=0,1,2, \ldots$. Here the summation is defined as zero when $m=0$.

Case $3\left[6\right.$, p. 75]. $T(z)=\int_{-1}^{1}(1-x)^{\alpha}(1+x)^{\beta}(z-x)^{-1} d x, \alpha, \beta>-1$.

$$
\begin{aligned}
& \alpha=0 \quad \beta=0 \quad T(z)=\ln \frac{z+1}{z-1} \\
& \alpha=0 \quad \beta=1 \quad T(z)=(z+1) \ln \frac{z+1}{z-1}-2 \\
& \alpha=0 \quad \beta=2 \quad T(z)=(z+1)^{2} \ln \frac{z+1}{z-1}-2 z-4 \\
& \alpha=0 \quad \beta=3 \quad T(z)=(z+1)^{3} \ln \frac{z+1}{z-1}-2 z^{2}-6 z-\frac{20}{3} \\
& \alpha=0 \quad \beta=4 \quad T(z)=(z+1)^{4} \ln \frac{z+1}{z-1}-2 z^{3}-8 z^{2}-\frac{38}{3} z-\frac{32}{3} \\
& \alpha=\frac{1}{2} \quad \beta=\frac{1}{2} \quad T(z)=\pi z-\pi(z-1)^{1 / 2}(z+1)^{1 / 2} \\
& \alpha=-\frac{1}{2} \quad \beta=-\frac{1}{2} \quad T(z)=\pi /\left[(z-1)^{1 / 2}(z+1)^{1 / 2}\right] \\
& \alpha=\frac{1}{2} \quad \beta=-\frac{1}{2} \quad T(z)=\pi-\pi(z-1)^{1 / 2} /(z+1)^{1 / 2} \\
& \alpha=1 \quad \beta=1 \quad T(z)=\left(1-z^{2}\right) \ln \frac{z+1}{z-1}+2 z \\
& \alpha=\frac{3}{2} \quad \beta=\frac{3}{2} \quad T(z)=\pi\left[(z-1)^{1 / 2}(z+1)^{1 / 2}\right]^{3}-\pi z^{3}+\frac{3 \pi}{2} z .
\end{aligned}
$$

The Gauss-Jacobi rules on $-1 \leqslant x \leqslant 1$ corresponding to the special cases $\alpha=0$ or $\beta=0$ are frequently tabulated $[2, \mathrm{pp} .118-121]$ on $[0,1]$. For this reason we list the Hilbert transform in the form given in Case 2. All of the results in Sections $1-4$ then apply with $[-1,1]$ replaced by $[0,1]$.

In the above listing the branch cut for the complex natural logarithm and square root functions is taken to be the negative real axis. When computing $T(z)$ the standard FORTRAN library routines CLOG and CSQRT can conveniently be employed.

For $|z|>1$, it may be useful to compute $T(z)$ from the series 


$$
T(z)=\sum_{\nu \geqslant 0} I\left(x^{\nu}\right) z^{-\nu-1},
$$

provided the moments corresponding to $\omega$ are known.

Mathematics Department

The University of Georgia

Athens, Georgia 30602

1. L. V. KANTOROVITCH, "On approximate calculation of certain types of definite integrals and other applications of the method of selection of the singularities," Mat. Sb., v. 41, 1934, pp. $235-245$.

2. V. I. KRYLOV, Approximate Calculation of Integrals, Fizmatgiz, Moscow, 1959; Transl. by A. H. Stroud, Macmillan, New York, 1962. MR 22 \#2002; 26 \#2008.

3. J. McNAMEE, "Error-bounds for the evaluation of integrals by the Euler-Maclaurin formula and by Gauss-ty pe formulae," Math. Comp., v. 18, 1964, pp. 368-381. MR 32 \#3264.

4. W. E. SMITH \& J. N. LYNESS, “Applications of Hilbert transform theory to numerical quadrature," Math. Comp., v. 23, 1969, pp. 231-252. MR 40 \#5132.

5. F. STENGER, "Bounds on the error of Gauss-ty pe quadratures," Numer. Math., v. 8, 1966, pp. 150-160. MR 33 \#5120.

6. G. SZEG ̈̈, Orthogonal Polynomials, rev. ed., Amer. Math. Soc. Colloq. Publ., vol. 23, Amer. Math. Soc., Providence, R. I., 1959. MR 21 \#5029. 